\title{
Metabolically induced liver inflammation leads to NASH and differs from LPS- or IL-1 $\beta$-induced chronic inflammation
}

\author{
Wen Liang ${ }^{1,2}$, Jan H Lindeman ${ }^{3}$, Aswin L Menke ${ }^{4}$, Debby P Koonen ${ }^{5}$, Martine Morrison ${ }^{1}$, Louis M Havekes ${ }^{1,2}$, \\ Anita M van den Hoek ${ }^{1,6}$ and Robert Kleemann ${ }^{1,3,6}$
}

The nature of the chronic inflammatory component that drives the development of non-alcoholic steatohepatitis (NASH) is unclear and possible inflammatory triggers have not been investigated systematically. We examined the effect of non-metabolic triggers (lipopolysaccharide (LPS), interleukin-1 $\beta$ (IL-1 $\beta$ ), administered by slow-release minipumps) and metabolic dietary triggers (carbohydrate, cholesterol) of inflammation on the progression of bland liver steatosis (BS) to NASH. Transgenic APOE3*Leiden.huCETP (APOE3L.CETP) mice fed a high-fat diet (HFD) developed BS after 10 weeks. Then, inflammatory triggers were superimposed or not (control) for six more weeks. Mouse livers were analyzed with particular emphasis on hallmarks of inflammation which were defined in human liver biopsies with and without NASH. Livers of HFD-treated control mice remained steatotic and did not progress to NASH. All four inflammatory triggers activated hepatic nuclear factor- $\kappa \mathrm{B}$ (NF- $\kappa \mathrm{B}$ ) significantly and comparably ( $\geq 5$-fold). However, HFD + LPS or HFD + IL-1 $\beta$ did not induce a NASH-like phenotype and caused intrahepatic accumulation of almost exclusively mononuclear cells. By contrast, mice treated with metabolic triggers developed NASH, characterized by enhanced steatosis, hepatocellular hypertrophy, and formation of mixed-type inflammatory foci containing myeloperoxidase-positive granulocytes (neutrophils) as well as mononuclear cells, essentially as observed in human NASH. Specific for the metabolic inducers was an activation of the proinflammatory transcription factor activator protein-1 (AP-1), neutrophil infiltration, and induction of risk factors associated with human NASH, that is, dyslipidemia (by cholesterol) and insulin resistance (by carbohydrate). In conclusion, HFD feeding followed by NF- $\kappa$ B activation per se (LPS, IL-1 $\beta$ ) does not promote the transition from BS to NASH. HFD feeding followed by metabolically evoked inflammation induces additional inflammatory components (neutrophils, AP-1 pathway) and causes NASH.

Laboratory Investigation (2014) 94, 491-502; doi:10.1038/labinvest.2014.11; published online 24 February 2014

KEYWORDS: human NASH; inflammation; neutrophils; NF- $\kappa$ B; steatosis

Non-alcoholic fatty liver disease (NAFLD) is emerging as one of the most common liver disorders in modern societies. ${ }^{1}$ Its prevalence in the general population is strongly increasing together with obesity, dyslipidemia, and the metabolic syndrome. ${ }^{2}$ NAFLD encompasses a range of conditions associated with the accumulation of fat within liver cells. The most benign form of NAFLD is bland steatosis (BS), which is characterized by the accumulation of lipid droplets.
BS can remain stable for years and will not progress in many cases. ${ }^{1}$ In other cases BS does progress, leading to the development of non-alcoholic steatohepatitis (NASH), which is characterized by fat accumulation and inflammation. This condition is not benign and can further progress to liver fibrosis and cirrhosis with high rates of morbidity and mortality. ${ }^{3,4}$ The factors that trigger this transition from BS to NASH are unknown.

\footnotetext{
'The Netherlands Organization for Applied Scientific Research (TNO), Department of Metabolic Health Research, TNO Metabolic Health Research, Leiden, The Netherlands; ${ }^{2}$ Departments of Endocrinology and Cardiology, Leiden University Medical Center, Leiden, The Netherlands; ${ }^{3}$ Department of Vascular Surgery, Leiden University Medical Center, Leiden, The Netherlands; ${ }^{4}$ TNO-Triskelion, Zeist, The Netherlands and ${ }^{5}$ Department of Molecular Genetics, University Medical Center Groningen, University of Groningen, Groningen, The Netherlands

Correspondence: Dr R Kleemann, PhD, The Netherlands Organization for Applied Scientific Research (TNO), Department of Metabolic Health Research, Zernikedreef 9 , 2333 CK Leiden, The Netherlands.

${ }^{6}$ These authors contributed equally to this work.

Received 6 November 2013; revised 17 January 2014; accepted 27 January 2014
} 
The pathogenesis of NASH is thought to be driven by a lipid component and an inflammatory component. ${ }^{2,5}$ Excessive intrahepatic fat accumulation may sensitize the liver to subsequent inflammatory insults that promote the development of NASH. The exact nature of these inflammatory insults or 'second hits,' viz the mechanism inducing the inflammatory component of the disease, is largely unclear. $^{6}$ The proinflammatory transcription factor nuclear factor- $\kappa \mathrm{B}(\mathrm{NF}-\kappa \mathrm{B})$ may critically influence this process and chronic activation of NF- $\kappa \mathrm{B}$ is associated with many pathogenic liver conditions. ${ }^{7}$ A causal role for hepatic $\mathrm{NF}-\kappa \mathrm{B}$ in the progression of BS to NASH has been proposed recently in transgenic mice selectively expressing constitutively active IKK $\beta$ in hepatocytes. ${ }^{8}$ Among the inflammatory triggers that may be responsible for chronic activation of NF- $\kappa \mathrm{B}$ in liver cells are circulating endotoxins (lipopolysaccharide (LPS)) or proinflammatory cytokines such as interleukin-1 $\beta$ (IL-1 $\beta$ ). ${ }^{9,10}$ However, low-grade hepatic inflammation may also be evoked by other types of stimuli such as unhealthy diets or excess eating, which represent metabolic triggers of inflammation that may activate multiple pathways simultaneously. ${ }^{11,12}$ For instance, diets with a high content of metabolizable energy from carbohydrate or diets rich in cholesterol can cause chronic activation of NF- $\kappa$ B in the liver. ${ }^{6,12-15}$

In this study, we tested the influence of different inflammatory triggers with NF- $\kappa \mathrm{B}$-activating properties on the transition of BS to NASH and analyzed whether disease progression depends on the type of trigger used. More specifically, we compared the effects of non-dietary inflammatory stimuli (LPS, IL-1 $\beta$ ) and diet-related metabolic inflammatory stimuli (carbohydrate, cholesterol), all of which were superimposed on a high-fat diet (HFD) in separate groups of mice. Transgenic APOE $3^{\star}$ Leiden.huCETP (E3L.CETP) mice were used because their lipoprotein metabolism is translational to the human situation and these animals are prone to develop obesity, dyslipidemia, and NAFLD on HFD. ${ }^{16,17}$ Chronic exposure to low concentrations of LPS and IL- $1 \beta$ was achieved by minipump technology, and metabolically evoked inflammation was induced by feeding diets rich in carbohydrates or cholesterol. Before analysis of experimental NASH, human liver biopsies with and without NASH were analyzed to define hallmarks of inflammation of human NASH. Combined histological and biochemical analysis of mouse livers resulted in the identification of cellular and molecular determinants that are crucial for the transition of BS to NASH.

\section{MATERIALS AND METHODS Human Liver Biopsies}

Human liver biopsies were obtained at autopsy for postmortem histological analysis by pathologists (Department of Pathology, Leiden University Medical Center (LUMC), Leiden, The Netherlands). $N=21$ specimens with NASH and $n=12$ control subjects without NASH were used for this study (diagnosis by pathologist). Tissue was obtained and handled in accordance with the guidelines set by the LUMC medical ethical committee. Cardiovascular disease was the predominant cause of death. In all, $40 \%$ of the subjects were female and $60 \%$ male. The average age was 62 years. Cross-sections were stained with anti-myeloperoxidase (MPO) (A0398; Dako, Glostrup, Denmark) and phosphop65-NF- $\kappa$ B (no. 3037; Cell Signaling, Danvers MA, USA) as described. ${ }^{18}$

\section{Animals and Diets}

Male APOE3L.CETP mice were 10-14 weeks old and obtained from an in-house breeding colony (TNO Metabolic Health Research). ${ }^{18}$ Briefly, ApoE ${ }^{\star} 3$-Leiden mice were crossed with hemizygous human CETP transgenic mice and the offspring was genotyped using the primers: CETP-F sequence (5'-GAATGTCTCAGAGGACCTCCC-3') and CETP-R sequence (5'-CTTGAACTCGTCTCCCATCAG-3'); APOC1-F sequence (5'-GGTCCCGGGCACTTCCCTTAGCCCCA-3') and APOC1-R sequence (5'-TTTGAGCTCGGCTCTTGAGACAGGAA-3'). Furthermore, the presence of human ApoE3 protein and human Cetp protein in the plasma was confirmed by ELISA.

Experiments were approved by an ethical committee on Animal care and Experimentation, Zeist, The Netherlands. Mice were kept on chow diet (Ssniff R/M-H; Ssniff Spezialdiäten, Soest, Germany) until the start of the experiment. Animals had free access to water and diet during the study period of 16 weeks. The group size was $n=9-10$. One group remained on chow and served as aging control. The other animals received an HFD (24\% (w/w) lard; Research Diets, New Brunswick, NJ, USA) for 10 weeks (run-in) and were then matched into groups based on total plasma cholesterol, triglycerides, and body weight. HFD feeding was continued in all groups. Groups were additionally treated with one of the following treatments until week 16: LPS from Salmonella minnesota R595 (lot. no. 30446A1; List-Biological Laboratories, Campbell CA, USA) by minipump at $5 \mu \mathrm{g}$ per day; recombinant murine IL-1 $\beta$ (lot. no. 030447; PeproTech, Rocky-Hill, NJ, USA) by minipump at 100 ng per day; dietary carbohydrate (Ensure ${ }^{\circledR}$ Plus in drinking water; Abbott Laboratories, Hoofddorp, The Netherlands); dietary cholesterol (1\% (w/w) mixed into HFD) (Sigma-Aldrich, Zwijndrecht, The Netherlands). Osmotic minipumps (lot. no. 10194-08; Alzet, Maastricht, The Netherlands) were placed subcutaneously in the back region (flow rate: $0.10 \mu \mathrm{l} / \mathrm{h}, 101 \mu \mathrm{l}$ total volume) under isoflurane anesthesia. Two controls groups were included, and they were treated with either HFD + PBS (minipump) or HFD only. These groups were comparable in all histological scores and were therefore analyzed as one.

\section{Histological and Biochemical Analysis}

The right median liver lobe (lobus dexter medialis hepatis) was carefully isolated, fixed in $4 \%$ paraformaldehyde ( 2 days), embedded in paraffin, and cross-sectioned $(5 \mu \mathrm{m})$. 
The other liver lobes were snap-frozen and stored at $-80^{\circ} \mathrm{C}$. Hematoxylin-eosin (HE)-stained cross-sections were scored blindly by a pathologist using an adapted grading method for human NASH, ${ }^{19}$ with specific emphasis on hallmarks of steatosis and inflammation. Briefly, two cross-sections were examined per mouse and the level of vacuolization was determined relative to the total liver area analyzed, that is, expressed as a percentage. The extent of vacuolization was scored as 'Slight' $(<5 \%)$, 'Moderate' (5-33\%), 'Severe' (34-66\%), and 'Very Severe' (>66\%). Of note, hepatocellular ballooning (central nucleus, web-like structure in the cytoplasm) is a hallmark of human NASH, but mouse livers merely show enlarged hepatocytes containing small intracellular lipid droplets, herein referred to as 'hepatocellular hypertrophy.' The extent of enlarged hepatocytes (diameter $>1.5$ normal) was analyzed using the same percentage categories as for vacuolization to provide quantitative information about abnormally enlarged cells. Inflammation was scored by counting and analyzing the type of inflammatory cells (mononuclear and/or polymorph nuclear) in a defined area of five random microscopic fields per animal. Inflammation was graded as 'Normal' ( $<0.5$ foci), 'Slight' (0.5-1.0 foci), 'Moderate' (1.0-2.0 foci), and 'Severe' ( $>2.0$ foci). Collagen was stained using Picro-Sirius red staining (SigmaAldrich).

\section{Liver Lipids}

The intrahepatic concentration of free cholesterol, triglycerides, and cholesteryl esters was analyzed as described. ${ }^{20}$ Briefly, $200 \mu \mathrm{g}$ of frozen liver biopsies were homogenized in MilliQ $\mathrm{H}_{2} \mathrm{O}$ and the protein content was determined. Two micrograms of cholesterol acetate was added to each sample as an internal standard. Lipids were extracted using methanol and chloroform. Extracted lipids were then separated by thinlayer chromatography using a silica-gel-60 precoated plate. Plates were incubated at $130{ }^{\circ} \mathrm{C}$ for $30 \mathrm{~min}$ and band densities were then quantified. ${ }^{20}$ In addition, cryostat sections were stained with Oil Red O (Sigma-Aldrich) to identify lipids, and counterstained with hematoxylin (Sigma-Aldrich) to visualize nuclei.

\section{Immunohistochemical Staining}

Paraffin-embedded liver cross-sections were used for the analysis of MPO-positive cells (neutrophils) using anti-MPO (ab9535; Abcam, Cambridge, UK). After pre-treatment with target retrieval solution (3-in-1; Dako) at pH 9.0, cross-sections were incubated with primary anti-MPO overnight. Sections were peroxidase blocked in $3 \% \mathrm{H}_{2} \mathrm{O}_{2}$ in methanol for $10 \mathrm{~min}$ and detected with Dako Envision-Flex amplification kit (Product no. K800021). For the analysis of CD11b, frozen liver samples were used. After fixation with acetone, cross-sections were incubated with anti-CD11b (lot. no. ab8878; Abcam) for $1 \mathrm{~h}$ followed by incubation with biotinylated rabbit anti-rat antibody (lot. no. 00065538; Dako). Immunoreactivity was visualized with streptavidin/HRP (lot. no. 00057815; Dako) and AEC (lot. no. 10043262; Dako). For all IHC analyses, negative controls were performed by omitting the primary antibody.

\section{Analysis of Transcription Factor Activity in Liver Homogenates}

To determine the amount of activated p65-NF- $\kappa \mathrm{B}$, activator protein-1 (AP-1), signal transducer and activator of transcription 3 (STAT3), and CCAAT/enhancer-binding protein$\beta$ (C/EBP- $\beta$ ) protein in livers, protein extracts were prepared using a Nuclear Extract Kit (cat. no. 40010; ActiveMotif, Rixensart, Belgium). The protein content of the extracts was determined with Bio-Rad dye reagent (cat. no. 500-0006; Bio-Rad Laboratories GmbH, Munich, Germany). Six micrograms of protein was used for transcription factor activity analysis using TransAM ${ }^{\circledR}$ kits for NF- $\kappa$ B-p65 Chemi, c-Jun (AP-1), STAT3, and C/EBP- $\beta$ kits (cat. nos. 40097, 46096, 45196, and 44196; ActiveMotif) as reported. ${ }^{11,20}$ Briefly, the amount of active transcription factor was determined by measuring its binding capacity to a consensus binding sequence in the presence of a competitor oligonucleotide or a mutant (non-competitor) oligonucleotide to control for the specificity of DNA binding. Data are provided as relative units.

\section{Plasma Parameters, Lipoproteins, and Plasma Inflammatory Markers}

EDTA (Sarstedt, Numbrecht, Germany) plasma was collected after a 5-h fast (0800-1300 hours). Plasma levels of glucose (Instruchemie, Delfzijl, The Netherlands), insulin (Mercodia, Uppsala, Sweden), total cholesterol, and triglycerides (Roche Diagnostics, Almere, The Netherlands) were measured with commercial kits. Homeostasis model assessment (HOMA) index was calculated according to the formula: HOMA = fasting plasma glucose $(\mathrm{mM}) \times$ fasting plasma insulin $(\mathrm{ng} /$ $\mathrm{ml} / 22.5$. Lipoprotein profile analysis was performed with AKTA-FPLC. ${ }^{18}$ The plasma levels of E-selectin and SAA were determined by ELISA (R\&D-Systems, Abington, UK for E-selectin; Life Technologies, Bleiswijk, The Netherlands for SAA). Plasma alanine transaminase (ALAT) activity was measured using a Reflotron ${ }^{\mathbb{B}}$ kit (Roche Diagnostics).

\section{Statistical Analysis}

Statistical analyses were performed with the StatView software (SAS Institute, Cary, NC, USA), and a $P$-value $<0.05$ was considered statistically significant. Differences between groups were analyzed using Mann-Whitney non-parametric test for two independent samples. The data obtained from histological scoring of livers were analyzed using two-sided Fisher's exact test. All values shown represent means \pm s.e.m.

\section{RESULTS \\ Characterization of the Inflammatory Component in Human NASH}

HE-stained human tissue biopsies were analyzed for steatosis and inflammation. Livers of patients with NASH showed 
pronounced vacuolization compared with controls (Figures la and b). Quantitative analysis revealed that about $40 \%$ of the surface area was steatotic $(P<0.001$ versus control; Supplementary Material A), of which 35\% was macrovesicular steatosis and 5\% microvesicular steatosis. Hepatocellular ballooning was observed in NASH livers but not in controls (Figures 1a and b). Also, enlarged hepatocytes containing small lipid droplets were observed in NASH specimens only (Supplementary Material B). NASH livers were characterized by lobular inflammation, that is, an abundant presence of mononuclear cells and polymorphnuclear leukocytes (granulocytes) that formed foci (Figures la and b). Inflammatory cell foci contained CD68-positive cells of the monocytes/macrophage lineage (not shown) as well as granulocytes with MPO-positive immunoreactivity (neutrophils), a hallmark of human NASH (Figures 1c and d). NASH livers also showed a pronounced expression of
p65-NF- $\kappa$ B (in inflammatory foci and steatotic areas), while only a few p65-NF- $\kappa$ B-positive cells were observed in healthy control livers (Figures 1e and f). Fibrosis was present in most (73\%) human NASH specimens, and in none of the controls (Figures $1 \mathrm{~g}$ and $\mathrm{h}$ ).

\section{Effects of HFD Feeding and Addition of Inflammatory Triggers on the Development of Experimental NASH}

To gain insight into the nature of the inflammatory trigger that may underlie the development of BS into NASH, we evaluated the effects of different inflammatory stimuli superimposed on HFD feeding. Treatment with inflammatory stimuli was started after a 10 -week run-in period on HFD, which resulted in BS and moderate pericentral hepatocellular vacuolization (Supplementary Material C). Lobular inflammation was not observed after 10 weeks of HFD run-in.

\section{Control}
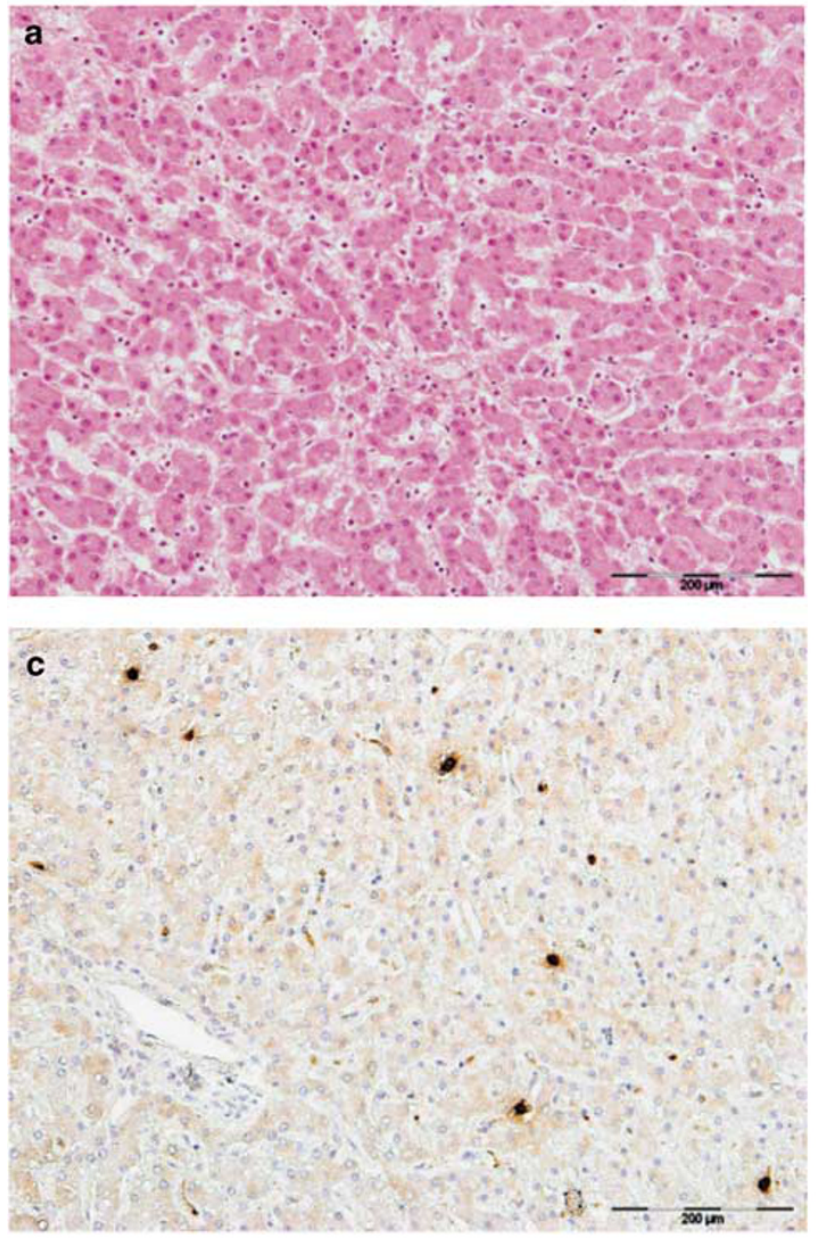
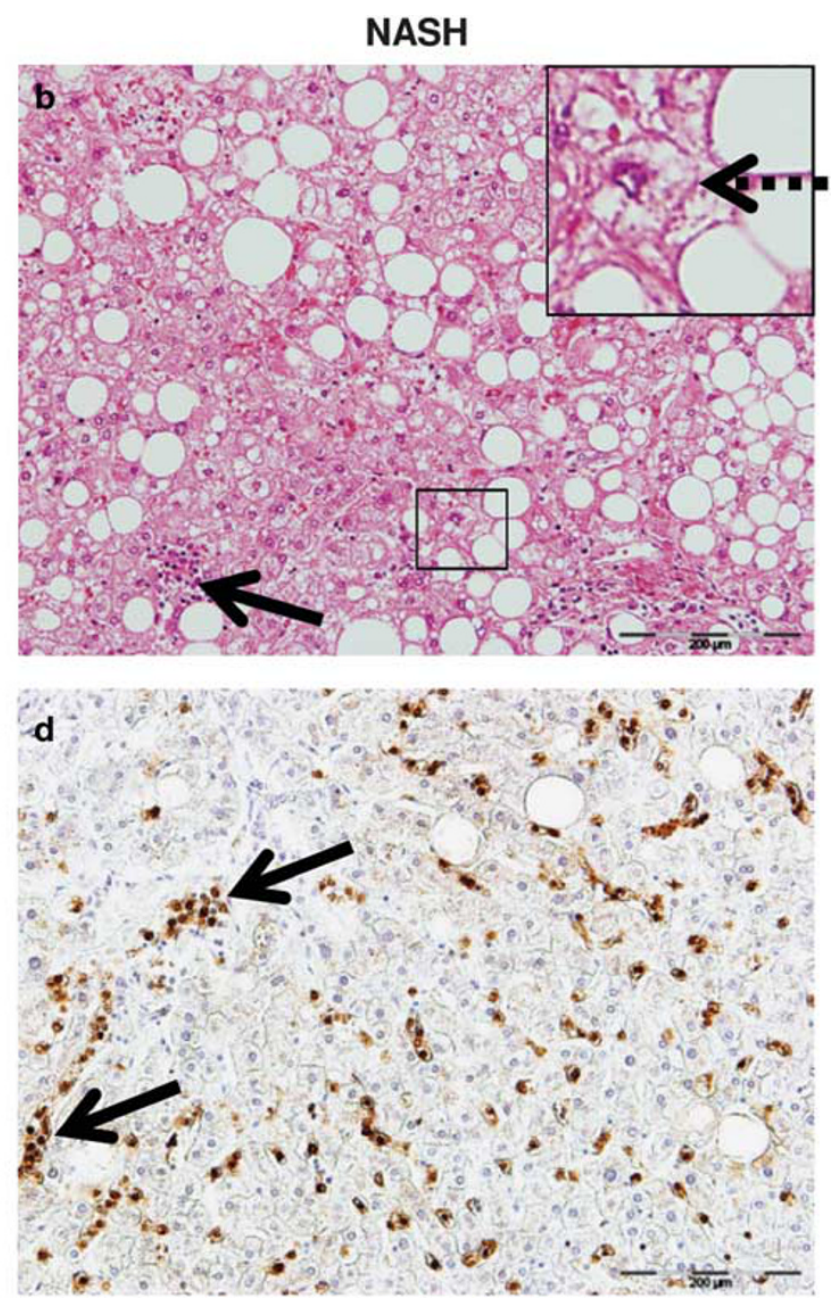

Figure 1 Analysis of inflammation in human non-alcoholic steatohepatitis (NASH). Representative photographs of control livers ( $n=12$, panels left) were compared with NASH specimens ( $n=21$, panels right). (a and b) Hematoxylin and eosin (HE) staining showed pronounced vacuolization in NASH livers, which was associated with inflammatory cell aggregates (solid arrow) and hepatocellular ballooning (magnified in the inset). (c and d) Immunostaining showed inflammatory cell foci (arrows) containing myeloperoxidase (MPO)-positive cells in NASH. (e and f) Immunostaining of p65-NF$\kappa \mathrm{B}$-positive cell clusters (arrows) in NASH (magnified in the inset). (g and $\mathbf{h}$ ) Picro-Sirius red staining demonstrated NASH with fibrosis in 15 out 21 specimen. NF- $\kappa \mathrm{B}$, nuclear factor- $\kappa \mathrm{B}$. 


\section{Control}
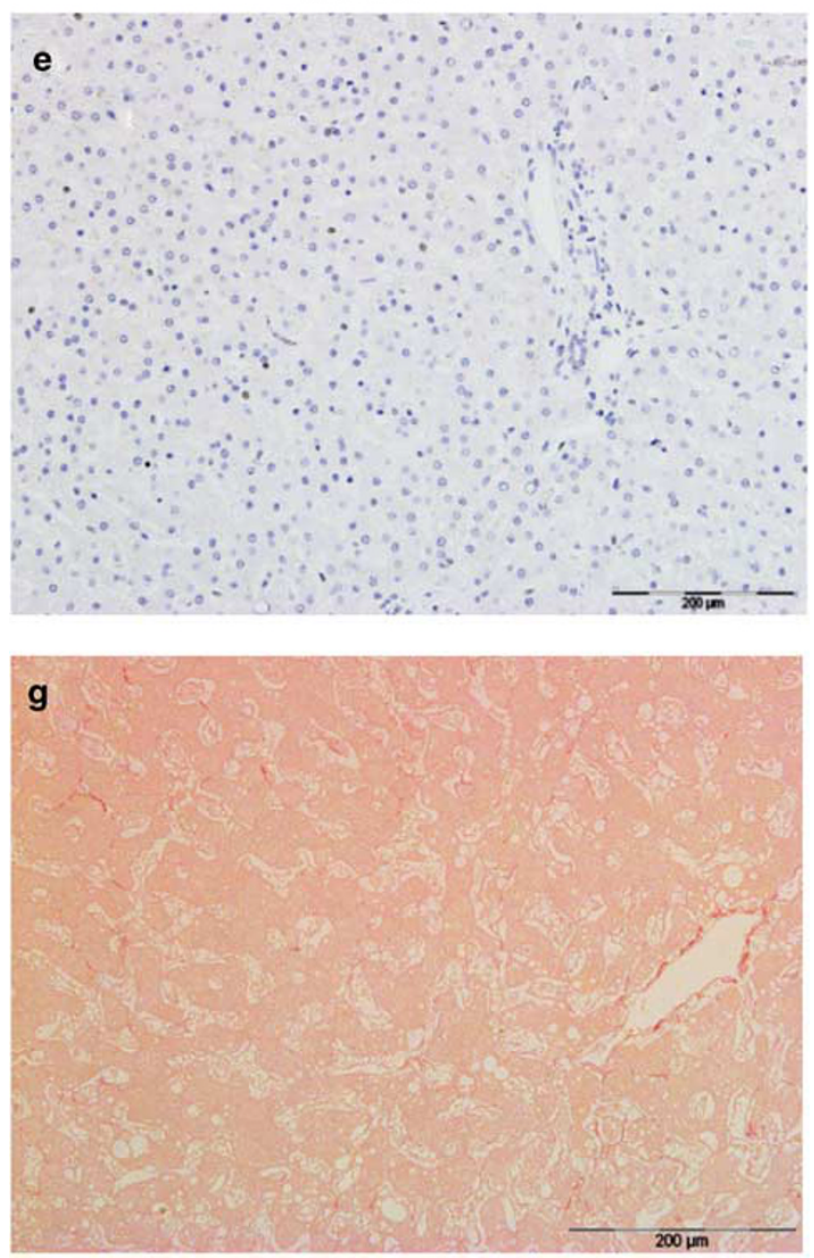
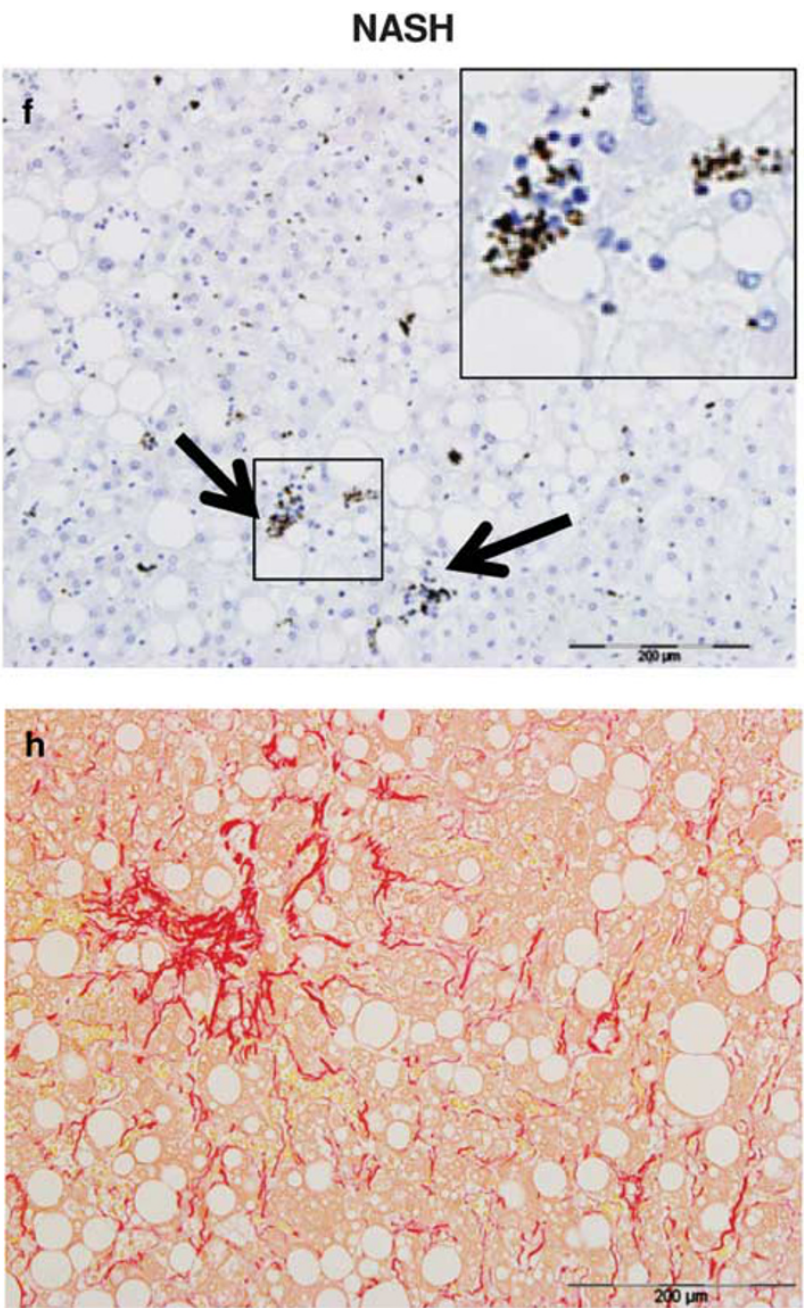

Figure 1 (Continued)

HFD feeding was continued for six more weeks and experimental groups additionally received either PBS (minipump control), LPS (minipump), IL-1 $\beta$ (minipump), dietary carbohydrate $\left(\right.$ Ensure $\left.^{\mathbb{R}}\right)$, or dietary cholesterol. A second control group was continued on HFD only. A separate group was fed chow during the complete experimental period (16 weeks) and served as a reference. All interventions were well tolerated and the superimposed inflammatory triggers did not affect the daily food intake relative to HFD control, that is, calorie intake was comparable between the groups (on average $12 \mathrm{kcal}$ per day per animal) except for the HFD + carbohydrate group ( $16 \mathrm{kcal}$ per day per animal). At the time of being killed, livers were immediately prepared for histological examination and biochemical analysis of active p65NF- $\kappa$ B. Representative photomicrographs of livers are shown in Figures 2a-f. Reference mice on chow had normal livers without steatosis or inflammation (Figure 2a). The two control groups, that is, HFD feeding alone and HFD + PBS (minipump) treatment, were comparable and showed moderate steatosis with vacuolization of the pericentral zone and the midzone, as well as hepatocellular hypertrophy (individual data provided in Supplementary Material D). Livers of these groups hardly contained inflammatory foci, demonstrating that HFD feeding per se is not sufficient to induce a NASH-like phenotype within a period of 16 weeks (Figure 2b).

Consistent with the low abundance of cellular inflammation in HFD-treated mice, the level of transcriptionally active p65-NF- $\kappa$ B protein measured by TransAM ${ }^{\circledR}$ was very low $\left(1.1 \times 10^{5}\right.$ relative units) (Figure $\left.3 \mathrm{a}\right)$. Treatment with the inflammatory triggers LPS, IL- $1 \beta$, carbohydrate, and cholesterol resulted in significantly higher levels of activated p65$\mathrm{NF}-\kappa \mathrm{B}$ protein in mouse livers. The p65-NF- $\kappa \mathrm{B}$-activating effects of the different inflammatory stimuli were statistically comparable. This demonstrates that p65-NF- $\kappa \mathrm{B}$ was activated at least fivefold or more in the liver at the end of the study relative to HFD alone.

Histological analysis of experimental NASH allowed quantification of the effects of the different inflammatory triggers on vacuolization, hypertrophy, and inflammation 

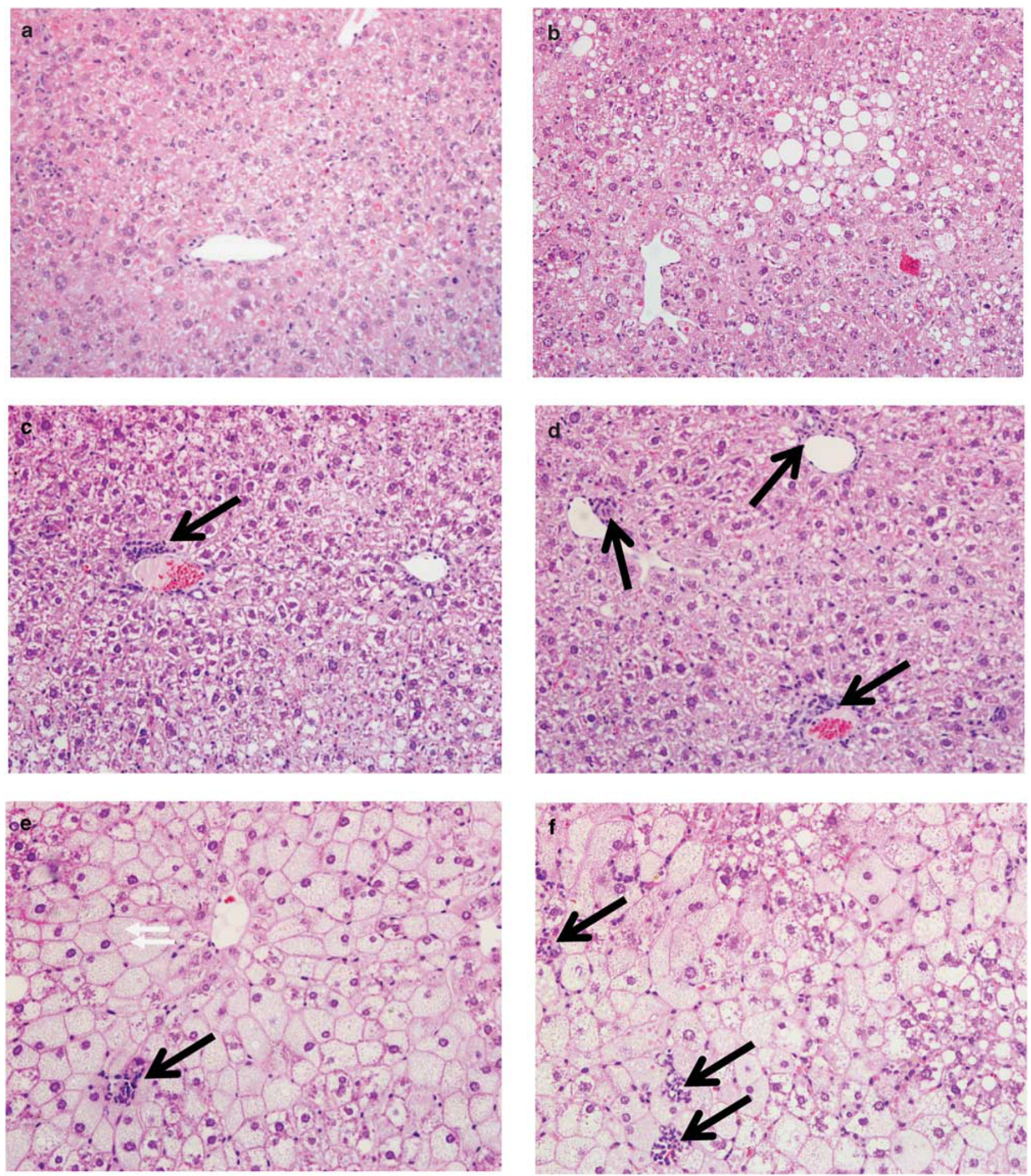

Figure 2 Histological presentation of experimental non-alcoholic steatohepatitis (NASH). Representative photomicrographs are shown at 200-fold magnification. Mice were treated with (a) chow (reference group), (b) high-fat diet (HFD) (control group) and HFD + additional inflammatory stimuli (c) lipopolysaccharide (LPS), (d) interleukin-1 $\beta$ (IL-1 $\beta$ ), (e) dietary carbohydrate, and (f) dietary cholesterol. Inflammatory infiltrates are indicated with arrows.

compared with HFD alone (Figures 3b-d; individual histological scores see Supplementary Materials D and E). Chronic exposure to LPS did not further aggravate vacuolization and hypertrophy relative to HFD control (Figures $2 c$ and $3 b, c)$.
The inflammatory cell content of LPS-treated animals was higher than in controls treated with HFD only. Inflammatory cells were mainly mononuclear and diffusely distributed, and polymorph nuclear cells were hardly observed. Surprisingly, 
a

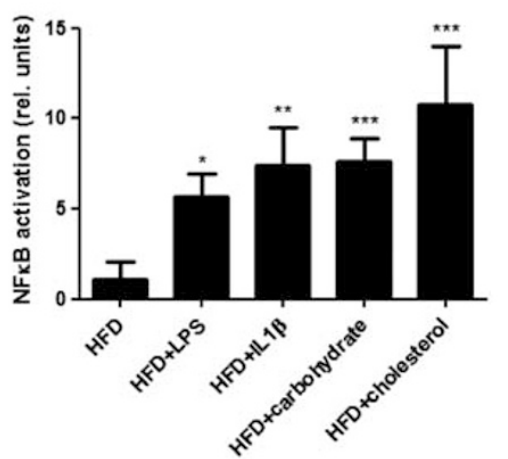

C
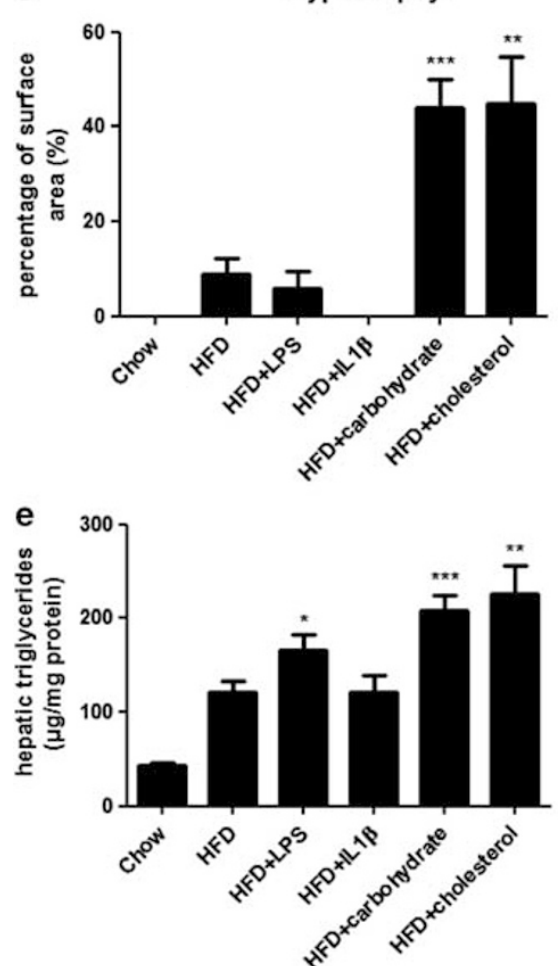

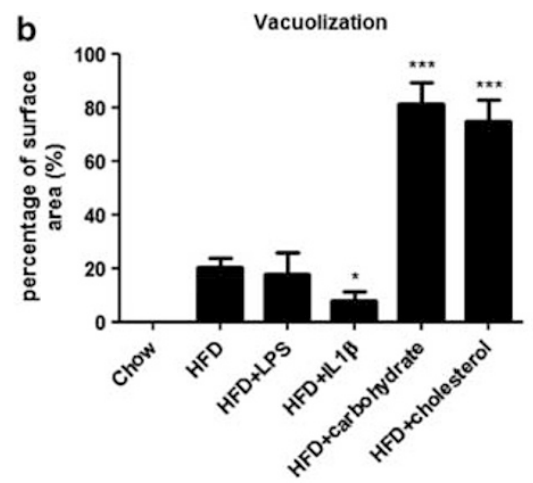

d

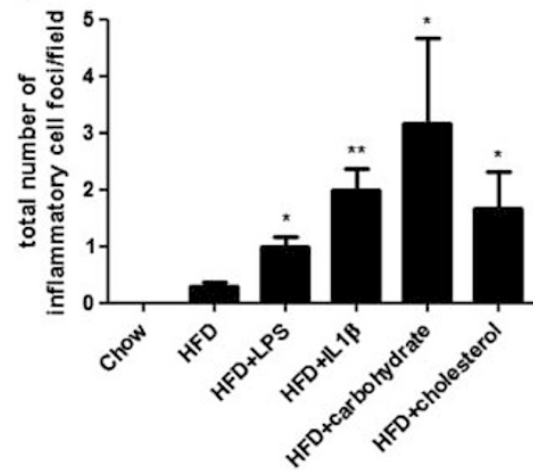

Figure 3 Quantitative analysis of experimental non-alcoholic steatohepatitis (NASH). Quantification of (a) activated p65-NF- $\kappa$ B measured by TransAM ${ }^{B}$ in liver homogenates, (b) vacuolization, and (c) hepatocellular hypertrophy as percentage of the total liver area analyzed (\%). (d) Number of inflammatory foci per microscopic field. (e) Quantitative analysis of the intrahepatic triglycerides. Groups treated with inflammatory stimuli are compared to high-fat diet (HFD) control. ${ }^{*} P<0.05$, ${ }^{* *} P<0.01$, and ${ }^{* * *} P<0.001$. NF- $\kappa \mathrm{B}$, nuclear factor- $\kappa \mathrm{B}$.

livers of mice treated with IL- $1 \beta$ showed even less vacuolization than HFD control mice (Figures $2 \mathrm{~d}$ and $3 \mathrm{~b}$ ). Hepatocellular hypertrophy was not observed with IL-1 $\beta$. Similar to LPS, the inflammatory cell content was higher than in HFD controls, but again inflammatory cells were predominately of the mononuclear type and diffusely distributed across the liver. Inflammatory cell foci were frequently found in close proximity to vascular structures.

In contrast to LPS and IL1 $\beta$, the metabolic stimuli (carbohydrate and cholesterol) clearly promoted the development of a NASH-like phenotype and resulted in pronounced changes in shape and size of hepatocytes: carbohydrate- treated animals showed pronounced microvacuolization and enlarged hepatocytes containing small lipid droplets, also in the periportal zone, which was not affected in HFD control livers (Figure 2e and Supplementary Material D). Quantification of hepatocellular vacuolization and hypertrophy revealed that carbohydrate feeding increased these parameters significantly and about fourfold $(P<0.05)$ relative to HFD (Figures $3 b$ and c). Mixed-type inflammatory cells (ie, mononuclear and polymorph nuclear cells) were present and inflammatory cell foci were observed.

Cholesterol-treated livers resembled carbohydrate-treated livers and also showed extensive micro- and macrovacuolization 

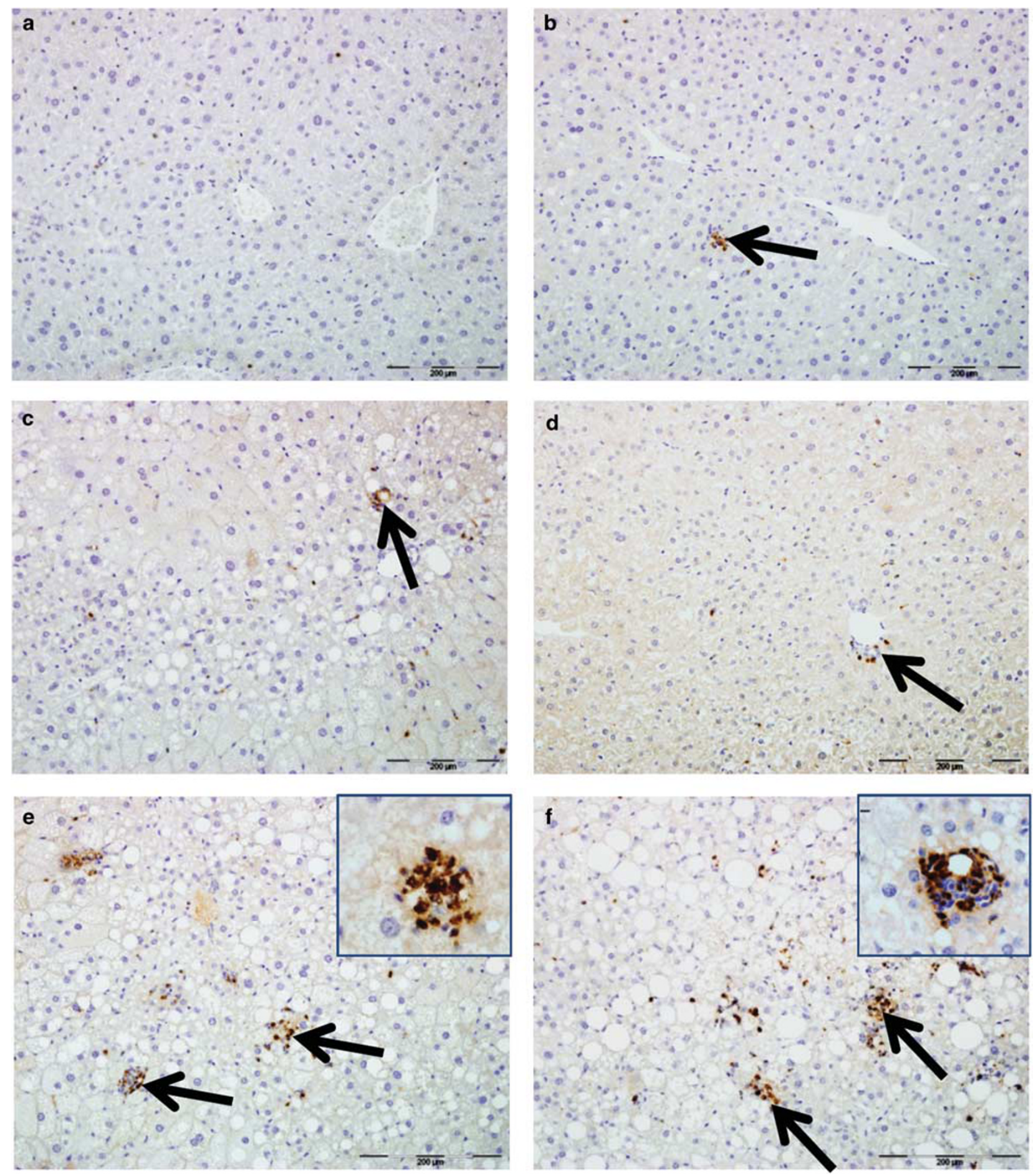

Figure 4 Characterization of inflammation in experimental non-alcoholic steatohepatitis (NASH). Immunohistochemical staining of myeloperoxidase (MPO) in (a) chow reference, (b) high-fat diet (HFD) control and HFD superimposed with (c) lipopolysaccharide (LPS), (d) interleukin-1 $\beta$ (IL-1 $\beta$ ),

(e) carbohydrate, or (f) cholesterol. Inflammatory cell foci (arrows) containing MPO-positive cells. (g) Relative contribution of mononuclear cell foci and mixed-type inflammatory cell foci to the overall content of inflammatory cells for each group. (h) Transcriptional activity of c-Jun (a subunit of activator protein 1 (AP-1)) protein by TransAM ${ }^{\mathbb{B}}$ analysis. (i) Lipoprotein profile at $t=16$ weeks. ${ }^{*} P<0.05$ and ${ }^{* *} P<0.01$.

with large vacuoles in pericentral and periportal zones. Livers had a significantly increased content of inflammatory cell foci (Figure 2f). The effects of cholesterol on vacuolization, hypertrophy, and inflammation were all significant (Figures 3b-d) and statistically comparable to the effect of the other metabolic trigger, carbohydrate.

Biochemical analysis of the intrahepatic triglyceride concentrations (Figure 3e) and parallel Oil Red O staining (not 

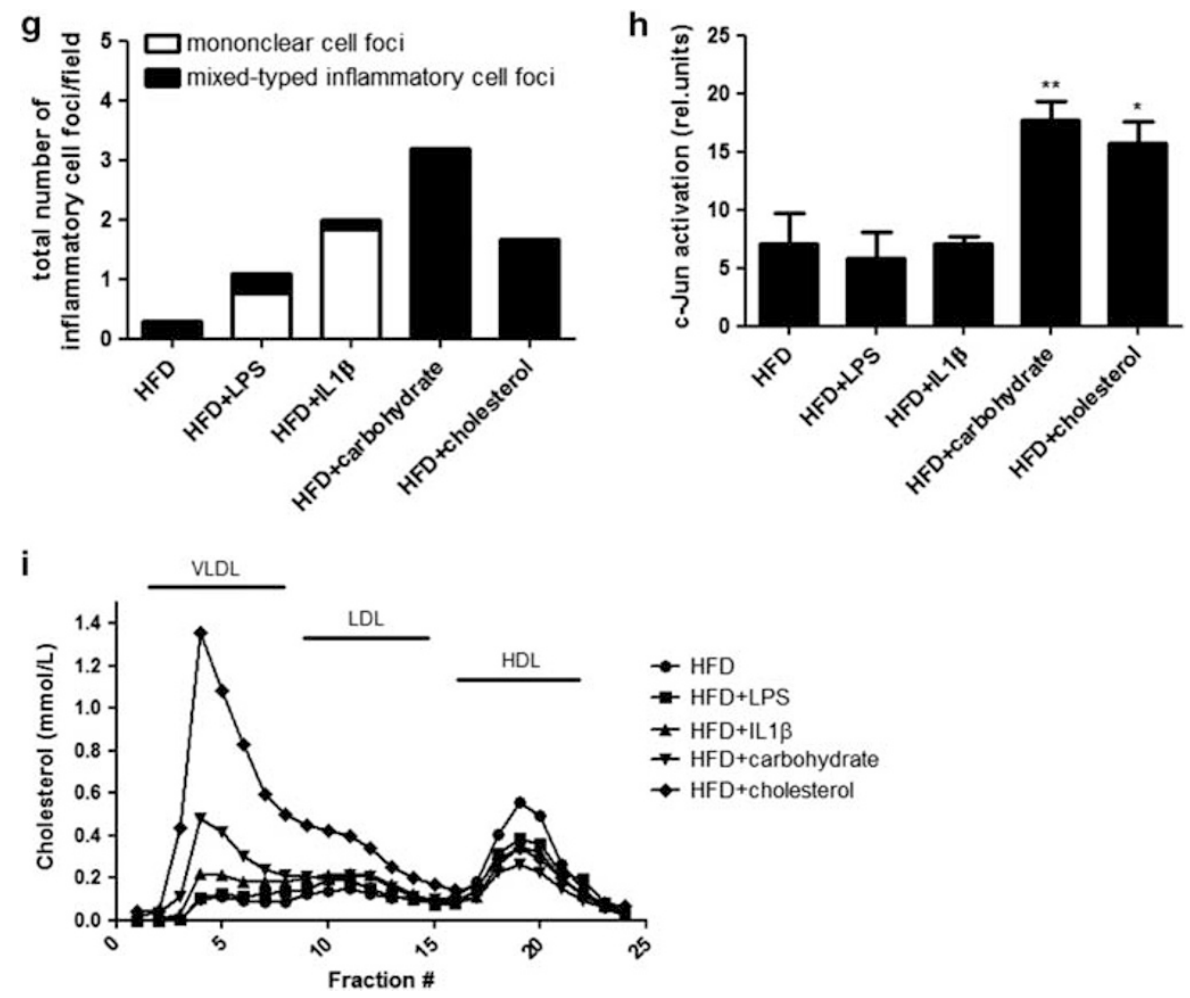

Figure 4 (Continued)

shown) were in line with the histological analyses and confirmed that only the metabolic triggers, carbohydrate and cholesterol, promoted a transition of BS to NASH. Most of the mice did not develop fibrosis within the study period: only one mouse (cholesterol-treated group) did develop NASH with fibrosis (Sirius red collagen staining, not shown).

\section{Effect of Non-Metabolic and Metabolic Inflammatory Triggers on Inflammatory Cells and Inflammatory Pathways}

Experimental livers were further analyzed histologically and biochemically to identify determinants that distinguish the metabolic triggers from LPS and IL-1 $\beta$. A more refined histological analysis of inflammatory cells present in the liver revealed that LPS and IL- $1 \beta$ treatment led to an almost exclusive recruitment of mononuclear cells, whereas carbohydrate- and cholesterol-treated livers contained mixed-type inflammatory cells including polymorph nuclear cells (Figure $4 \mathrm{~g}$ ). Consistent with this observation, more MPOpositive cells (neutrophils) were observed in the groups exposed to carbohydrate or cholesterol but not in those treated with LPS or IL-1 $\beta$ (Figures $4 \mathrm{a}-\mathrm{f}$ ). Immunohistochemical analysis of the monocyte/macrophage marker CD68 revealed no difference among the experimental groups, except a slight increase in the IL- $1 \beta$-treated (1.5-fold) and cholesterol-treated (2-fold) groups (not shown). Furthermore, the number of Mac1/CD11b-positive cells did not differ between the groups (not shown). Taken together, this shows that an abundant presence of neutrophils, a hallmark of human NASH, discriminates the two metabolic inflammatory triggers from the two non-dietary triggers LPS and IL- $1 \beta$.

To gain more insight into the molecular effects of the different triggers, we analyzed transcription factor activity downstream of specific inflammatory pathways by TransAM ${ }^{\circledR}$ technology. Independent of the type of inflammatory trigger, the activity of C/EBP- $\beta$ tended to be lower than in HFD controls (Supplementary Material F). The transcriptional activity of STAT3 was slightly increased in the LPS- and IL1 $\beta$ treated groups but the effects did not reach statistical significance Supplementary Material G). Treatment with carbohydrate and cholesterol significantly increased the transcriptional activity of c-Jun (AP-1), an effect that differentiated the metabolic triggers from LPS and IL- $1 \beta$ (Figure $4 \mathrm{~h}$ ). With respect to systemic markers of inflammation, plasma E-selectin levels were increased by IL- $1 \beta$ and plasma SAA levels were increased by IL- $1 \beta$, carbohydrate, and cholesterol treatment (Table 1).

\section{Effect of Non-Metabolic and Metabolic Inflammatory Triggers on Risk Factors Associated with NASH}

Table 1 shows data of risk factors that are typically associated with NASH development in humans, for example, body weight, visceral fat mass, plasma lipids, fasting glucose and insulin, and ALAT. These risk factors were not affected by LPS. IL-1 $\beta$ treatment also did not affect these parameters, except a slight increase in triglycerides and a decrease in 
Table 1 Experimental treatments have a differential effect on risk factors of NASH

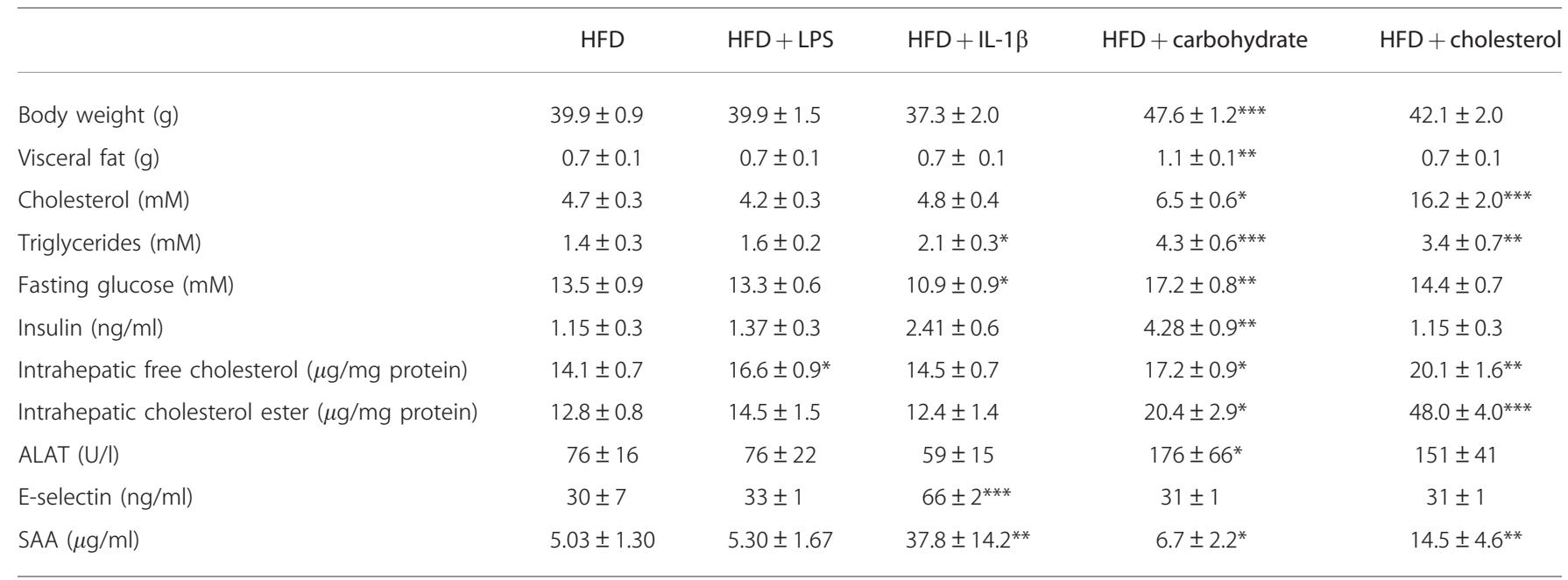

Parameters shown were determined in fasting plasma at the end of the experimental period (week 16). Intrahepatic lipids were determined in freshly prepared tissue homogenates. Values are means \pm s.e.m. Significance is indicated as ${ }^{*} P<0.05,{ }^{* *} P<0.01$, and ${ }^{* * *} P<0.001$ versus HFD control (Mann-Whitney test).

glucose. In contrast, the metabolic triggers worsened risk factors that are typically associated with NASH development in humans. Carbohydrate treatment resulted in an increased body weight $(47.6 \pm 1.2 \mathrm{~g} ; P<0.001)$ and visceral fat mass $(1.1 \pm 0.05 \mathrm{~g} ; P<0.01)$ beyond that of HFD controls. Also, plasma cholesterol and triglyceride concentrations were significantly higher in carbohydrate-treated animals. Increased fasting plasma glucose $(17.2 \pm 0.8 \mathrm{mM} ; P<0.01)$ and insulin $(4.28 \pm 0.9 \mathrm{ng} / \mathrm{ml} ; P<0.05)$ levels indicated insulin resistance $\left(\mathrm{HOMA}_{\text {carbohydrate }}=3.3\right.$ versus $\mathrm{HOMA}_{\mathrm{HFD}}$ control $\left.=0.9\right)$. Treatment with cholesterol had a marked adverse effect on plasma cholesterol and triglycerides and little effect on the other risk factors. Also, intrahepatic free cholesterol and cholesterylester levels were increased. Lipoprotein profile analysis revealed that the observed increase in plasma cholesterol was confined to VLDL/LDL-sized particles. All treatment groups showed a relative decrease in HDL compared with HFD control animals (Figure 4i). Plasma ALAT levels were only elevated in the groups treated with metabolic inflammatory triggers.

\section{DISCUSSION}

There is ample evidence that development of NASH is driven by chronic inflammation but the nature of the inflammatory component is not very clear, ${ }^{1-5,21}$ and possible inflammatory triggers have not been investigated systematically. The present study examined the influence of metabolically evoked inflammation (carbohydrate, cholesterol) and non-metabolic inflammatory triggers (LPS, IL-1 $\beta$ ) on the development of experimental NASH. Herein, we present the first head-to-head comparison of these triggers, revealing that they differ in their potency to stimulate progression of steatosis and induction of NASH. Only the metabolic triggers aggravated steatosis and led to an infiltration of neutrophils as well as activation of
AP-1, a hallmark of lipotoxicity. ${ }^{22}$ Furthermore, they induced metabolic risk factors (insulin resistance, dyslipidemia) associated with NASH development in humans, which were not observed for the non-metabolic triggers.

The HFD control group that remained on HFD during the complete experimental period did not show inflammatory cell infiltrates, had low AP- 1 and NF- $\kappa$ B levels, and did not progress from steatosis to NASH. Treatment with metabolic or non-metabolic inflammatory triggers activated hepatic NF- $\kappa \mathrm{B}$ significantly and comparably. Despite this clear proinflammatory effect in the liver, LPS- and IL- $1 \beta$-treated mice did not progress to NASH. Thus, simultaneous HFD feeding and activation of hepatic NF- $\kappa \mathrm{B}$ per se in livers with established steatosis is not sufficient to induce NASH development, indicating that additional inflammatory factors or pathways are involved. Livers of LPS- and IL1 $\beta$-treated groups exhibited cell infiltrates consisting almost exclusively of mononuclear cells, with hardly any mixed-type infiltrates, a characteristic feature of biopsy-proven human NASH. ${ }^{21}$ The selective effect of endotoxin on recruitment of mononuclear cells is in line with human and rodent studies in which elevated LPS activity in the serum was associated with macrophage-mediated chronic inflammation and increased levels of monocyte chemoattractant protein-1.23,24 Thus, chronic LPS and IL- $1 \beta$ exposure may merely activate and recruit specific populations of inflammatory cells to the liver and the absence of neutrophil infiltrates indicates that these triggers by themselves do not cause hepatocellular damage. Consistent with this notion, we observed no increase in ALAT when mice were treated with LPS or IL-1 $\beta$. Hence, administration of LPS and IL- $1 \beta$ by minipump constitutes an 'exogenous' form of inflammation, while the two metabolic triggers employed herein affect liver metabolism ${ }^{20}$ and 'endogenous' inflammatory processes related to it, ${ }^{12,14}$ 
possibly causing liver damage. Lipotoxicity and/or the relatively high levels of free cholesterol observed in these groups may constitute causes of liver damage.

Neutrophil infiltration is an important hallmark of biopsyproven NASH in humans ${ }^{21}$ that distinguishes subjects with NASH from those without, independent of obesity. ${ }^{25}$ It is not clear whether neutrophils are innocent bystanders or causally involved in the pathogenesis, and investigators have only recently begun to characterize the role of neutrophils in NAFLD. The molecular mechanisms that allow neutrophils to home to the liver are also not well understood. ${ }^{26} \mathrm{We}$ observed a marked infiltration of neutrophils with metabolic triggers despite low E-selectin levels. In accordance with our observations, adhesion of neutrophils within liver sinusoids is thought to be independent of selectins and differs fundamentally from other tissues. ${ }^{26}$ The activation of AP-1 may explain why metabolic inflammatory triggers stimulate neutrophil recruitment: the main chemoattractants for neutrophils in mice, keratinocyte cytokine (CXCL-8/IL-8 in humans) and macrophage inflammatory protein-2 (MIP-2), are transcriptionally regulated by NF- $\kappa \mathrm{B}$ and AP- $1 .{ }^{27}$ Transcription of MIP-2 depends on a simultaneous activation of both transcription factors, that is, a condition that was achieved with two metabolic triggers in the present study, but was absent in the case of the two non-metabolic triggers. Further support for a crucial role of the AP-1 signaling pathway in NASH comes from knockout studies: phosphorylation of the AP-1 subunit c-Jun is diminished in Jnk1-deficient mice, and these mice show a reduced development of steatohepatitis. ${ }^{28}$

Of note, the two metabolic triggers induced different factors that are known to be risk factors for NASH in humans, that is, adiposity/insulin resistance (carbohydrate) and dyslipidemia (cholesterol). This suggests that the pathogenic processes leading to liver damage, neutrophil infiltration, and AP-1 activation may differ between these triggers. Because plasma levels of HDL were reduced by both metabolic as well as non-metabolic triggers, it is unlikely that HDL has a major role in the pathogenic processes mediating the transition from steatosis to NASH. Our data also do not indicate a major role for the inflammatory pathways leading to the activation of STAT3 or C/EBP- $\beta$.

Treatment with inflammatory stimuli was started once steatosis was already manifest (after 10 weeks of HFD feeding) and reportedly still reversible, ${ }^{29}$ to mimic a risk population with established steatosis. ${ }^{21,30}$ HFD feeding alone causes metabolic stress and renders livers susceptible to injury ${ }^{13,20}$ and the superimposed insults represent 'second hits.' Importantly, feeding of an HFD alone only resulted in a steatotic liver within the study period and did not cause NASH or cellular inflammation. This has also been observed in other HFD-feeding studies of comparable lengths of time using diets with similar content of fat, that is, $\leq 25 \%$ (w/w). This equals about $45 \%$ energy from fat, ${ }^{20}$ which is reached in human diets in Finland and Crete. ${ }^{31}$ Of note, experimental diets that contain very high, supraphysiological quantities of fat (eg, $>50 \%$ energy) as well as experimental diets deficient in methionine and choline (MCD diets) may develop a more severe and different liver pathology. The translational character of such studies is debated because MCD-fed mice lose weight owing to a vastly lower caloric intake and do not become insulin resistant, while most humans with NASH are obese and insulin resistant. ${ }^{1-3}$

It is generally assumed that chronic activation of NF- $\kappa \mathrm{B}$ in the liver is a main driver of the transition from BS to NASH. In the present study, we compared different NF- $\kappa \mathrm{B}$-inducing inflammatory triggers (head-to-head and superimposed on HFD) regarding their ability to induce this transition to NASH. We observed that activation of NF- $\kappa \mathrm{B}$ in the liver per se (upon stimulation with LPS or IL-1 $\beta$ ) does not promote a transition from BS to NASH. By contrast, metabolic inducers of inflammation like cholesterol and carbohydrate had a greater ability to induce a human NASH-like phenotype. This suggests that chronic inflammation caused by metabolic triggers activates additional inflammatory pathways and biological processes than LPS or IL-1 $\beta$. To our knowledge, this is the first study that compares metabolic inflammatory triggers (high carbohydrate, cholesterol) to non-metabolic (classical) inflammatory chronic triggers (IL-1 $\beta$, LPS) in a head-to-head approach, and that defines molecular differences between 'metabolic' and 'non-metabolic' inflammatory triggers. More specifically, we show that metabolic triggers activate AP-1 in the liver and lead to neutrophil infiltration, both of which could be causative factors in the development of NASH. These mechanistic insights as well as the experimental conditions defined herein may help to further elucidate the etiology of NASH and contribute to the development of therapeutic strategies directed at attenuating metabolic overload and associated pathologic conditions.

Supplementary Information accompanies the paper on the Laboratory Investigation website (http://www.laboratoryinvestigation.org)

\section{ACKNOWLEDGMENTS}

We thank Erik H Offerman, Niels Kloosterhuis, José WA van der Hoorn, Adri Mulder, Karin Toet, and Simone van der Drift-Droog for technical assistance. This research was performed within the framework of CTMM, the Center for Translational Molecular Medicine (http: //www.ctmm.nl/), project PREDICCt (Grant No. 01C-104), and supported by the Dutch Heart Foundation, Dutch Diabetes Research Foundation, and Dutch Kidney Foundation. It was also financially supported by the TNO research program 'Personalized Prevention and Therapy-Systems Medicine'.

\section{DISCLOSURE/CONFLICT OF INTEREST}

The authors declare no conflict of interest.

1. Bellentani S, Marino M. Epidemiology and natural history of nonalcoholic fatty liver disease (NAFLD). Ann Hepatol 2009;8(Suppl 1):S4-S8.

2. Edmison J, McCullough AJ. Pathogenesis of non-alcoholic steatohepatitis: human data. Clin Liver Dis 2007;11:75-104, ix.

3. Lazo M, Clark JM. The epidemiology of nonalcoholic fatty liver disease: a global perspective. Semin Liver Dis 2008;28:339-350. 
4. Fujii H, Kawada N. Inflammation and fibrogenesis in steatohepatitis. J Gastroenterol 2012;47:215-225.

5. Harmon RC, Tiniakos DG, Argo CK. Inflammation in nonalcoholic steatohepatitis. Expert Rev Gastroenterol Hepatol 2011;5:189-200.

6. Nomura K, Yamanouchi T. The role of fructose-enriched diets in mechanisms of nonalcoholic fatty liver disease. J Nutr Biochem 2012; 23:203-208.

7. Wieckowska A, Feldstein AE. Diagnosis of nonalcoholic fatty liver disease: invasive versus noninvasive. Semin Liver Dis 2008;28:386-395

8. Cai D, Yuan M, Frantz DF, et al. Local and systemic insulin resistance resulting from hepatic activation of IKK-beta and NF-kappaB. Nat Med 2005;11:183-190.

9. Ruiz AG, Casafont F, Crespo J, et al. Lipopolysaccharide-binding protein plasma levels and liver TNF-alpha gene expression in obese patients: evidence for the potential role of endotoxin in the pathogenesis of non-alcoholic steatohepatitis. Obes Surg 2007;17: 1374-1380.

10. Nozaki Y, Saibara T, Nemoto $Y$, et al. Polymorphisms of interleukin-1 beta and beta 3-adrenergic receptor in Japanese patients with nonalcoholic steatohepatitis. Alcohol Clin Exp Res 2004;28(Suppl Proc):106S-110SS.

11. Liang W, Tonini G, Mulder $\mathrm{P}$, et al. Coordinated and interactive expression of genes of lipid metabolism and inflammation in adipose tissue and liver during metabolic overload. PLoS One 2013;8:e75290.

12. Kleemann R, Verschuren L, van Erk MJ, et al. Atherosclerosis and liver inflammation induced by increased dietary cholesterol intake: a combined transcriptomics and metabolomics analysis. Genome Biol 2007;8:R200.

13. Wielinga PY, Yakala GK, Heeringa $P$, et al. Beneficial effects of alternate dietary regimen on liver inflammation, atherosclerosis and renal activation. PLoS One 2011;6:e18432.

14. Vergnes L, Phan J, Strauss M, et al. Cholesterol and cholate components of an atherogenic diet induce distinct stages of hepatic inflammatory gene expression. J Biol Chem 2003;278:42774-42784.

15. Neuschwander-Tetri BA. Hepatic lipotoxicity and the pathogenesis of nonalcoholic steatohepatitis: the central role of nontriglyceride fatty acid metabolites. Hepatology 2010;52:774-788.

16. Bijland S, van den Berg SA, Voshol PJ, et al. CETP does not affect triglyceride production or clearance in $\mathrm{APOE}^{*} 3$-Leiden mice. J Lipid Res 2010;51:97-102.

17. Westerterp M, van der Hoogt CC, de Haan W, et al. Cholesteryl ester transfer protein decreases high-density lipoprotein and severely aggravates atherosclerosis in $\mathrm{APOE}^{*} 3$-Leiden mice. Arterioscler Thromb Vasc Biol 2006;26:2552-2559.

18. Lindeman $\mathrm{JH}$, Abdul-Hussien $\mathrm{H}$, van Bockel $\mathrm{JH}$, et al. Clinical trial of doxycycline for matrix metalloproteinase- 9 inhibition in patients with an abdominal aneurysm: doxycycline selectively depletes aortic wall neutrophils and cytotoxic T cells. Circulation 2009;119:2209-2216.

19. Kleiner DE, Brunt EM, Van Natta M, et al. Design and validation of a histological scoring system for nonalcoholic fatty liver disease. Hepatology 2005;41:1313-1321.

20. Kleemann $R$, van Erk M, Verschuren $L$, et al. Time-resolved and tissuespecific systems analysis of the pathogenesis of insulin resistance. PLoS One 2010;5:e8817.

21. Hubscher SG. Histological assessment of non-alcoholic fatty liver disease. Histopathology 2006;49:450-465.

22. Van Rooyen DM, Gan LT, Yeh MM, et al. Pharmacological cholesterol lowering reverses fibrotic NASH in obese, diabetic mice with metabolic syndrome. J Hepatol 2013;59:144-152.

23. Lassenius MI, Pietilainen $\mathrm{KH}$, Kaartinen $\mathrm{K}$, et al. Bacterial endotoxin activity in human serum is associated with dyslipidemia, insulin resistance, obesity, and chronic inflammation. Diabetes Care 2011;34:1809-1815.

24. Westerterp M, Berbee JF, Pires NM, et al. Apolipoprotein C-I is crucially involved in lipopolysaccharide-induced atherosclerosis development in apolipoprotein E-knockout mice. Circulation 2007;116:2173-2181.

25. Rensen SS, Slaats Y, Nijhuis J, et al. Increased hepatic myeloperoxidase activity in obese subjects with nonalcoholic steatohepatitis. Am J Pathol 2009;175:1473-1482.

26. Kubes $P$, Mehal WZ. Sterile inflammation in the liver. Gastroenterology 2012;143:1158-1172.

27. Orlichenko LS, Behari J, Yeh TH, et al. Transcriptional regulation of CXC-ELR chemokines KC and MIP-2 in mouse pancreatic acini. Am J Physiol Gastrointest Liver Physiol 2010;299:G867-G876.

28. Singh $R$, Wang $Y$, Xiang $Y$, et al. Differential effects of JNK1 and JNK2 inhibition on murine steatohepatitis and insulin resistance. Hepatology 2009;49:87-96.

29. Radonjic M, Wielinga PY, Wopereis S, et al. Differential effects of drug interventions and dietary lifestyle in developing type 2 diabetes and complications: a systems biology analysis in LDLr - / - mice. PLoS One 2013;8:e56122.

30. Brunt EM. Pathology of nonalcoholic fatty liver disease. Nat Rev Gastroenterol Hepatol 2010;7:195-203.

31. Hu FB, Manson JE, Willett WC. Types of dietary fat and risk of coronary heart disease: a critical review. J Am Coll Nutr 2001;20:5-19. 